\title{
Actif sur plusieurs terrains
}

\section{Bruno Kesseli}

Dr med. et lic. phil., rédacteur en chef

\author{
L'assemblée plénière annuelle a une nouvelle fois offert un large aperçu des activités \\ et des champs d'action de l'Institut suisse pour la formation médicale postgraduée et \\ continue (ISFM).
}

En sa qualité de centre de compétences pour toutes les questions en lien avec la formation postgraduée et continue, l'ISFM a des échanges permanents avec les autres acteurs de ce domaine: sociétés de discipline médicale, universités ou l'Office fédéral de la santé publique (OFSP). C'est certainement le signe de bonnes relations si le président de l'ISFM Werner Bauer a ouvert la séance plénière sur un bref éloge de la collaboration avec Catherine Gasser, docteur en droit et responsable de la division Professions médicales à l'OFSP; une personnalité, à ses yeux "compétente, communicative et apte au consensus», qui se lance dans un nouveau défi professionnel et qui, de ce fait, était présente pour la dernière fois - au moins dans sa fonction à l'OFSP - à cette séance plénière. Avant de lui remettre un petit cadeau d'adieu, Werner Bauer lance un léger clin d'œil à Catherine Gasser en déclarant n'avoir ressenti que très rarement chez elle cette "satisfaction du fonctionnaire qui dirige».

\section{Flash d'information}

\section{MedEd Symposium, ISFM-Award, promotion de projets}

A l'instar d'un "flash d'information", le président a fait le tour d'horizon des multiples activités de l'ISFM et pré-
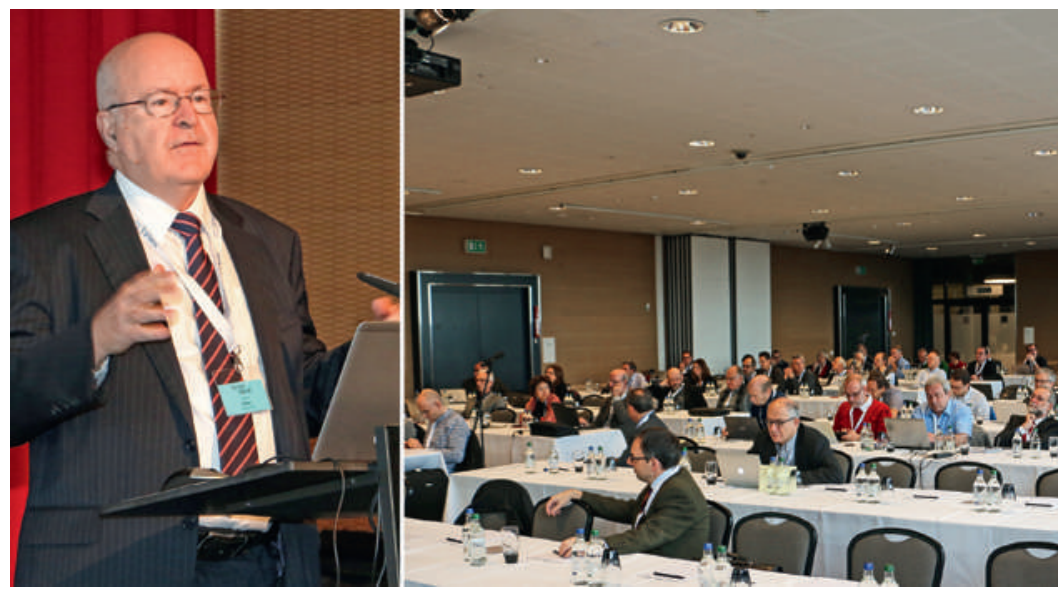

Un large éventail: Werner Bauer, président de I'ISFM, face à l'assemblée plénière de 2015. senté un bref aperçu des principaux thèmes et champs d'action de son institut, dont notamment le deuxième et très apprécié MedEd Symposium [1] et l'ISFM-Award, une distinction remise aux médecins cadres sur recommandation de deux de leurs anciens assistants [2]. Werner Bauer souligne que ce prix ne doit pas être (mal) interprété comme un classement mais bien comme la reconnaissance d'un engagement exceptionnel en faveur de la relève médicale. Doté d'un budget annuel de 125000 à $150000 \mathrm{CHF}$, la promotion de projets de l'ISFM a aussi reçu un écho très favorable. En 2015, pas moins de 23 projets ont été soumis à un jury chargé de sélectionner ceux qui méritent d'être subventionnés. Les projets retenus (cf. encadré à la page suivante) seront présentés lors du MedEd Symposium 2016.

\section{Plate-forme "Avenir de la formation médicale»} L'ISFM participe à la plate-forme "Avenir de la formation médicale» qui réunit 17 organisations partenaires sous la présidence de l'OFSP dans le but de plancher sur la mise en œuvre de la loi sur les professions médicales (LPMéd) et d'élaborer des solutions aux différents problèmes. Un modèle pour estimer le besoin en spécialistes de demain a été développé par le groupe thématique "Coordination de la formation postgrade des médecins» co-présidé par Werner Bauer. Une étude pilote, prévue en 2016, testera

\section{Assemblée plénière de I'ISFM}

Organe autonome de la FMH, I'Institut suisse pour la formation médicale postgraduée et continue (ISFM) réunit tous les acteurs et organismes principaux du domaine de la formation médicale postgraduée et continue, et garantit aux médecins une formation de qualité élevée dans plus de 120 domaines spécialisés. L'assemblée plénière annuelle de I'ISFM remplit différentes fonctions. D'une part, les membres du plénum forment un comité doté d'un pouvoir de décision et d'élection, qui traite les affaires relevant de son domaine de compétences à l'instar de la Chambre médicale de la FMH. De l'autre, I'assemblée, à laquelle sont également conviés des invités de provenances diverses, constitue une plate-forme chargée de transmettre des informations sur des thèmes spécifiques, de garantir l'échange d'expérience entre les participants et de débattre de toutes sortes de sujets. 


\section{Promotion de projets 2016}

Les projets suivants sont subventionnés par I'ISFM (150000 CHF au total):

- Développement et implémentation d'une plate-forme électronique générique pour le Workplace Based Assessment - e-WPBA (Hôpital cantonal de Bâle)

- Une application pour améliorer l'apprentissage de l'auscultation cardiopulmonaire par les médecins-assistants (CHUV, Service de médecine interne; EPFL)

- Introduction d'un feed-back multi-source dans la formation médicale prégraduée et postgraduée (Hôpital cantonal de Winterthur)

la fiabilité du modèle et donnera lieu en 2017 à un rapport d'appréciation sur la base duquel un organisme encore à constituer pourrait éventuellement formuler des recommandations en vue de cibler la formation postgraduée en fonction du besoin. En décembre 2015, la plate-forme a convoqué un autre groupe thématique placé sous la direction de l'OFSP et de l'association des hôpitaux H+ et consacré à la "Spécialisation croissante en médecine humaine sous l'angle de l'organisation hospitalière».

\section{Attestation de formation complémentaire «Echographie focalisée»}

Werner Bauer a précisé les réflexions qui ont conduit le Comité de l'ISFM à se prononcer l'année dernière en faveur de la création d'une attestation de formation complémentaire "Echographie focalisée». L'ultrason est une méthode d'examen de plus en plus utilisée dans le monde entier pour des questions médicales définies. Il est donc important d'en garantir la bonne qualité. C'est ce que fait cette AFC, qui n'a en aucun cas été conçue dans le sens d'une formation au rabais. Il est également prévu de pouvoir approfondir la formation «focalisée» et, dans une deuxième phase de formation, d'obtenir l'attestation de formation complémentaire globale en ultrasonographie.

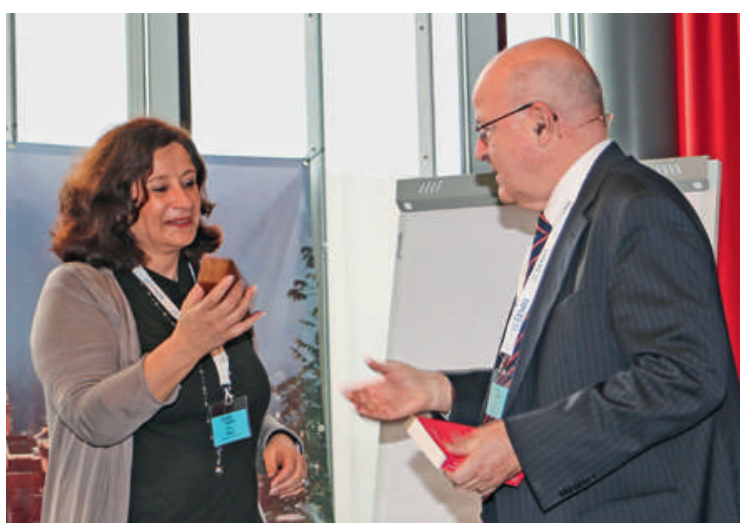

Collaboration constructive: Werner Bauer remet un petit cadeau d'adieu à Catherine Gasser.

\section{Objectifs de formation généraux}

Interrogés dans le cadre d'une enquête lancée par la direction de l'ISFM sur les objectifs de formation généraux, les responsables d'établissements de formation postgraduée reconnus ont identifié des faiblesses principalement dans les domaines de la gestion des conflits, de la communication, de l'économie et la médecine et du leadership. Un enseignement ciblé de ces matières dès le début de la formation pourrait avoir des effets positifs mais devrait aussi faire partie intégrante du quotidien des hôpitaux et des établissements de formation. Une autre possibilité de combler ces lacunes serait de proposer des cours dans les grands centres et en périphérie. Dans ce domaine, l'ISFM pourrait prêter son concours notamment en proposant des coaches ou du matériel didactique.

Organisés en collaboration avec le Royal College of Physicians of London, les ateliers "Teach the teachers" ont suscité un écho très positif. Ils auront de nouveau lieu au printemps et à l'automne prochains (pour les dates, cf. encadré).

\section{Elections et budget 2016}

Le mandat de Werner Bauer arrivera à son terme en 2016 mais il est disposé à se représenter à la présidence afin de permettre la transition avant sa succession. Son élection se déroulera dans le cadre de la Chambre médicale alors que le successeur du vice-président, Hans-Rudolf Koelz, dont le mandat se termine également cette année, sera élu par l'assemblée plénière de l'ISFM.

Le budget 2016 de l'ISFM, qui prévoit une perte d'environ 700000 CHF, a été approuvé par la Chambre médicale. Les provisions à hauteur de 6 millions de francs constituées avant 2013 ont dû être utilisées en 2014 pour couvrir les pertes, liées principalement aux projets informatiques, dont notamment le dispendieux logbook électronique. Les mesures adoptées pour réduire les coûts des projets informatiques devraient produire leurs

\footnotetext{
Teach the teachers

Ateliers 2016 du Royal College of Physicians et de I'ISFM à I'Hôpital cantonal de Lucerne:

- 19 avril Teaching in clinical settings

- 20 avril A masterclass on effective teaching skills

- 21/22 avril Effective strategies for feedback, assessment and supporting underperforming trainees

- 13 septembre Teaching in clinical settings

- 14 septembre Supporting the underperforming trainee

15/16 septembre Leadership for doctors

Inscription et plus amples informations:

mihriye.habermacher[at]luks.ch, tél. 0412055102.
} 
effets d'ici 2018. Mais d'autres baisses des dépenses sont étudiées. La discussion porte également sur les différentes possibilités d'augmenter les recettes. Le Comité a déjà décidé d'étendre l'obligation de taxe à tous les diplômes de formation continue figurant dans le registre des médecins.

\section{RFP, règlement, titres, LPMéd}

Conformément à la Réglementation pour la formation postgraduée (RFP) révisée, il est possible depuis 2016 de créer des "formations approfondies interdisciplinaires» pouvant être obtenues en complément à un titre de spécialiste. Il s'agit notamment de cursus de formation postgraduée qui correspondent à un profil spécifique de la profession de médecin et permettent de l'exercer en tant qu'activité principale. Au cours de la journée, le plénum a décidé la création de la première formation approfondie interdisciplinaire en "médecine palliative» (cf. ci-après).

$\mathrm{Au}$ premier janvier 2016, une autre modification de la RFP est entrée en vigueur: désormais, pour la reconnaissance d'un titre obtenu à l'étranger, tout spécialiste doit nécessairement justifier de deux ans de formation postgraduée en Suisse.

La fusion de la SSMG et de la SSMI a donné corps à la SSMIG et impliqué une modification de règlement. La nouvelle société de discipline sera représentée à partir de 2016 par deux délégués au sein du Comité de l'ISFM. La FMH et l'ISFM ont élaboré des recommandations concernant la mention de dénominations académiques, de titres de spécialiste, d'affiliation ou autres qualifications médicales. Elles sont aujourd'hui publiées et accessibles en ligne [3].

Lors de la révision de la loi sur les professions médicales (LPMéd), les Chambres fédérales ont décidé qu'il serait obligatoire de maîtriser une langue nationale pour exercer la médecine en Suisse. Elles ont également décidé une obligation d'enregistrement de tous les médecins, indépendants ou salariés, qui exercent une activité clinique. Ces modifications devraient prendre effet dans le courant de l'année.

\section{Stratégie de l'ISFM}

La stratégie de l'ISFM est à concevoir en relation avec les huit idéaux de la formation médicale postgraduée, que Werner Bauer a brièvement rappelés, entre autres: transfert de compétences, motivation, importance du Teaching, financement, évaluation et orientation en fonction du besoin. Pour les organes dirigeants de l'ISFM, la stratégie ne constitue pas une ligne directrice rigide et figée mais un instrument qu'il s'agit de réévaluer régulièrement et d'adapter si nécessaire. Les défis ne manquent pas comme en témoigne la longue liste des dossiers en suspens, évoquée par Werner Bauer, qui va du financement à la durée de la formation postgraduée, en passant par la question des plans de carrière, les nouvelles méthodes médicales, le besoin en médecins, et se termine sur des projets spécifiques tels que le logbook électronique et la plate-forme de formation continue, pour ne citer que les principaux. L'impact des changements structurels dans les hôpitaux (par ex. centres interdisciplinaires, nouvelle répartition des domaines, départements externalisés) sur la situation en matière de formation postgraduée constitue également une problématique de plus en plus importante. Le président invite le plénum à y réfléchir et à apporter des solutions aux problèmes qui s'annoncent.

\section{Révision du catalogue suisse des objectifs d'apprentissage}

La révision de ce catalogue se déroule sous l'acronyme PROFILES pour Principal relevant objectives and framework for integrated learning and education in Switzerland. Lors de son exposé, Pierre-André Michaud nous a permis de visualiser quelques-uns des principaux domaines dans lesquels travaille la commission chargée de la révision et placée sous sa direction. Après la phase d'un curriculum essentiellement scientifique (1900) et celle de l'apprentissage par problèmes (1960), nous sommes entrés aujourd'hui dans la troisième génération des concepts de formation médicale, caractérisée par une application des compétences professionnelles clés dans des contextes spécifiques. Dans ce concept, une place centrale est réservée aux EPA (Entrustable professional activities) qui ne sont autres que des aptitudes professionnelles si bien maîtrisées qu'elles peuvent être exercées sans supervision. Fin janvier 2016, les facultés de

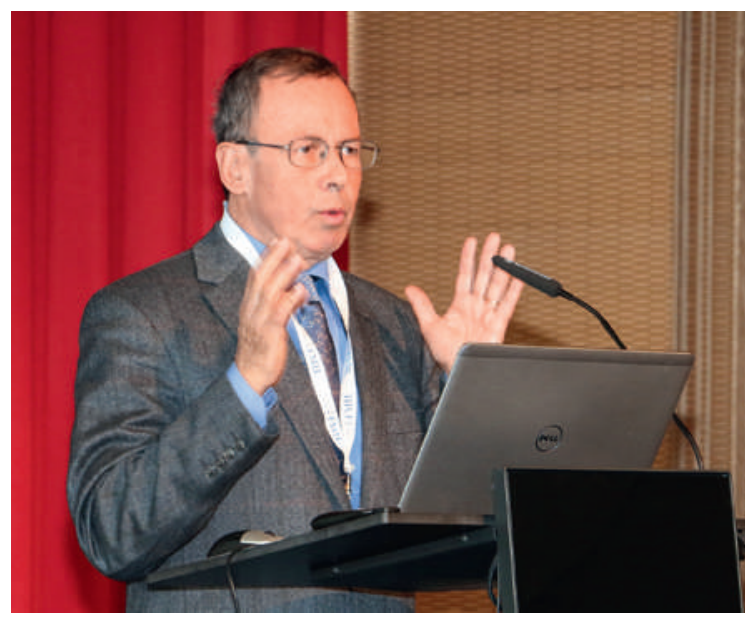

Focalisé: Pierre-André Michaud évoque la révision du catalogue suisse des objectifs d'apprentissage. 
médecine seront invitées à donner leur avis sur la première version des objectifs d'apprentissage prévus. Deux interventions après l'exposé ont cependant rappelé que les sociétés de discipline souhaitaient être associées à ces discussions. Werner Bauer s'est montré ouvert à ce point de vue.

\section{Accréditation 2018}

Le corps médical, avec ses structures de droit privé, a jusqu'à présent toujours organisé la formation postgraduée de manière autonome, ou du moins en grande partie autonome. Pour cela, il doit cependant obtenir une reconnaissance publique des programmes de formation postgraduée, de la Réglementation pour la formation postgraduée et aussi de l'ISFM en tant qu'organisation en charge de la formation, délivrée au terme d'une procédure d'accréditation avec le Département fédéral de l'intérieur (DFI). La procédure comprend notamment les demandes d'accréditation, des évaluations internes et externes, et les décisions. La dernière accréditation a eu lieu en 2011, la prochaine est prévue en 2018. L'ISFM informera les sociétés de discipline de la marche à suivre et des échéances.

\section{MEBEKO}

Commission extraparlementaire du DFI, chargée entre autres de donner son avis sur les demandes d'accréditation du domaine de la formation prégraduée et postgraduée, la Commission des professions médicales (ME$\mathrm{BEKO}$ ) remplit la fonction d'autorité et de conseil pour tout ce qui a trait aux professions médicales universitaires. Sa présidente Christina Kuhn Bänninger et le viceprésident Hans Hoppeler ont permis à l'auditoire de prendre connaissance des tâches de la commission et

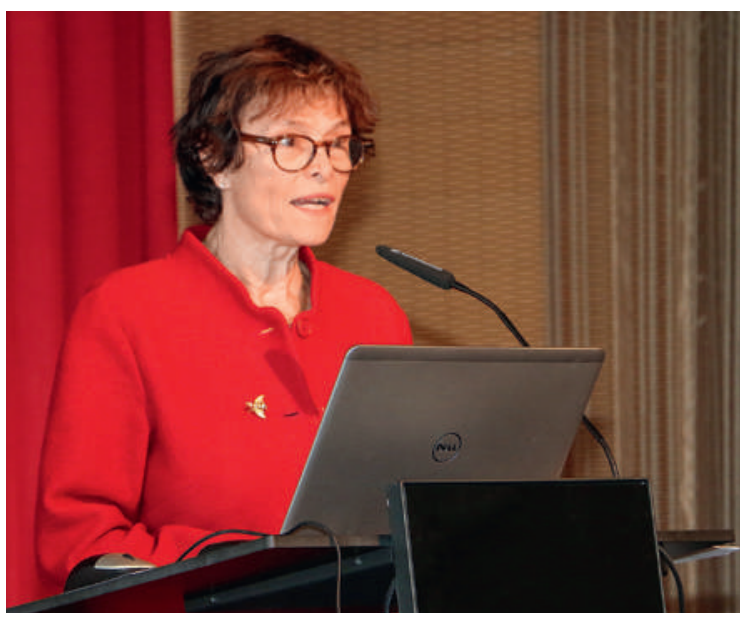

Regard sur les travaux de la commission: Christina Kuhn Bänninger, présidente de la MEBEKO.

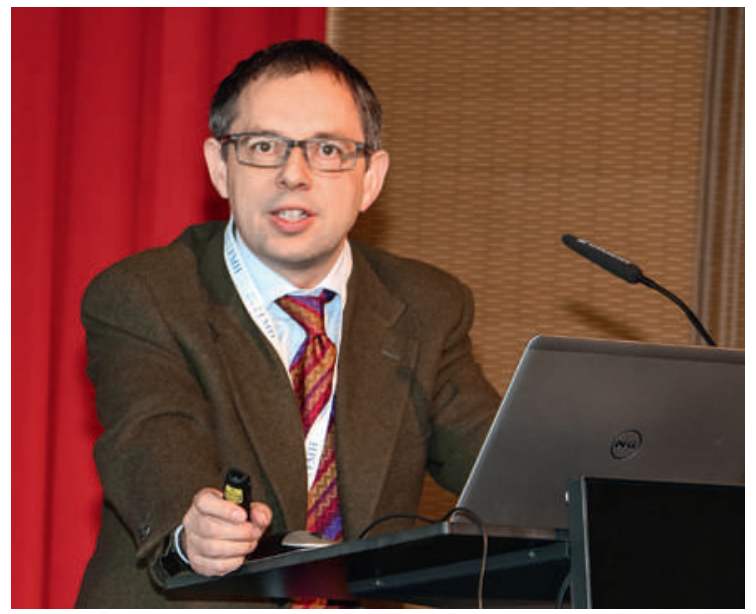

Michael Siegrist de l'EPFZ présente les résultats de l'enquête des médecins 2014/15.

d'en découvrir le rôle dans la procédure d'accréditation. La discussion qui s'en est suivie a soulevé des questions en lien avec le choix et la qualification des experts. Hans Hoppeler a rappelé que les sociétés de discipline avaient la possibilité de refuser les experts considérés comme inappropriés.

\section{Evaluation de la formation postgraduée}

L'évaluation de la formation postgraduée repose en partie sur une enquête auprès des médecins en formation réalisée chaque année par le département Health Sciences and Technology de l'Ecole polytechnique fédérale de Zurich. Michael Siegrist, Professeur de Consumer Behavior à l'EPFZ, a présenté les résultats de l'enquête 2014/15 [4]. De manière générale, les médecins en formation ont donné de bonnes notes, inversement proportionnelles à la grandeur des établissements de formation postgraduée, qui varient en moyenne entre 4,7 et 5,4 sur une échelle de 1 à 6 . Ils estiment que les objectifs de formation généraux sont importants et l'enquête relève un potentiel d'amélioration dans la manière de les enseigner. Il a été agréable de voir que les évaluations en milieu de travail (EMiT) ont été bien notées, tant par les premiers concernés, les médecins en formation, que par les responsables d'établissements, qui ont donné leur avis à ce sujet dans une enquête séparée. Un résultat surprenant pour Werner Bauer qui est plutôt habitué à entendre des critiques sur cet instrument d'évaluation.

\section{Formation approfondie}

Les délégués ont eu pour la première fois à se prononcer sur la création d'une formation approfondie interdisciplinaire proposée pour la médecine palliative. Comme Gian Domenico Borasio, Daniel Büche et Christoph Cina 


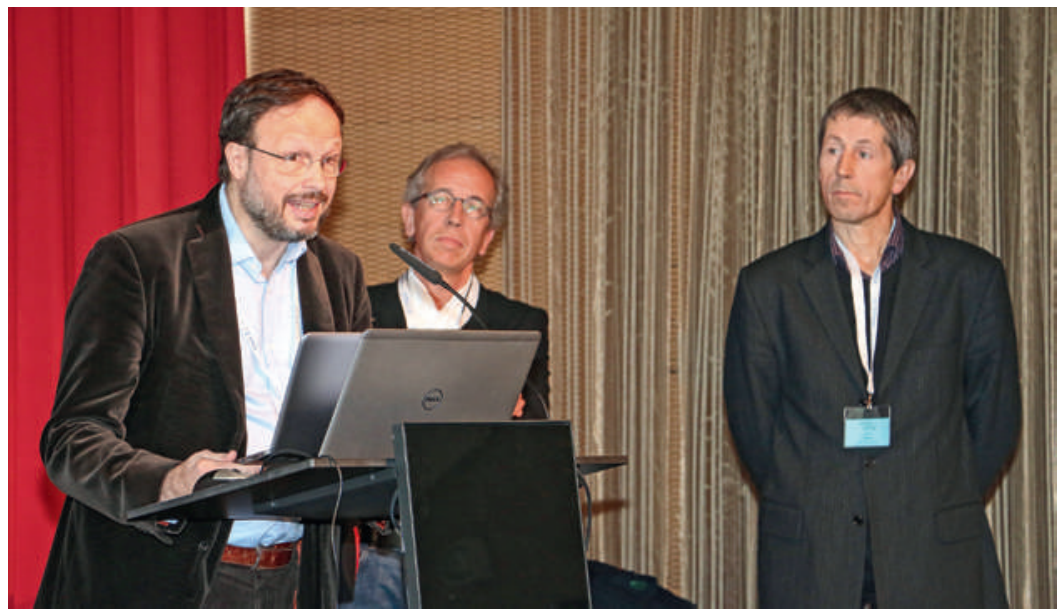

Engagés: Gian Domenico Borasio, Christoph Cina et Daniel Büche argumentent en faveur de la création de la formation approfondie interdisciplinaire en médecine palliative. l'ont expliqué, la durée et le contenu du cursus sont d'une ampleur significative et offrent la possibilité aux titulaires de cette qualification d'exercer une activité principale en médecine palliative. Du reste, un grand nombre de sociétés de discipline médicale soutient cette proposition. Après une brève discussion, qui a été l'occasion d'entendre quelques voix critiques, la création de la formation approfondie interdisciplinaire en médecine palliative a été approuvée sans opposition et neuf abstentions. C'est également sans opposition que la proposition d'une formation approfondie en "psychiatrie et psychothérapie des addictions» a été acceptée.

\section{Logbook électronique et plate-forme de formation continue}

Lorsqu'on évoque le logbook électronique, les superlatifs sont manifestement de rigueur: Werner Bauer a parlé de "mégaprojet» et, dans son état des lieux, Christoph Hänggeli a remercié tous les protagonistes pour l'«énorme tour de force». Entre-temps, tous les 82 programmes de formation postgraduée sont paramétrés, près de 10000 utilisateurs enregistrés et le cockpit destiné à la Commission des titres mis en service. Les demandes de titre et les plans de formation ne peuvent désormais être qu'exclusivement traités via le logbook électronique.

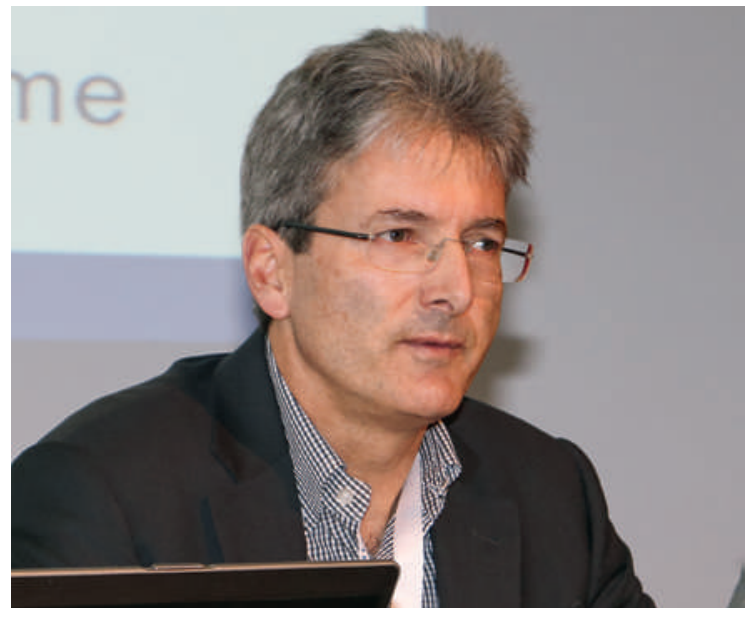

"Un énorme tour de force», déclare Christoph Hänggeli, directeur de I'ISFM, à propos du logbook électronique.

La plate-forme de formation continue permet aux médecins de gérer en ligne leur formation continue. S'ils remplissent les conditions requises, ils peuvent directement imprimer leur diplôme de formation continue qui apparaît automatiquement sur www.doctorfmh.ch et valide toutes les positions de droits acquis. Les délégués ont approuvé sans opposition la deuxième phase de ce projet qui vise à mettre en place la saisie en ligne des crédits et un registre centralisé des sessions de formation continue.

Le cru 2015 de la séance plénière a mis en exergue de manière tout à fait remarquable les nombreux et différents terrains sur lesquels agissait et devait agir l'ISFM. Si quelques-uns sont caillouteux, les efforts déployés portent souvent leurs fruits. En revanche, nous n'avons pas à craindre que le travail puisse manquer au sein de l'ISFM...

\section{Crédits photos \\ Bruno Kesseli}

\section{Références}

1 Kesseli B. Se préparer efficacement aux défis de demain. Deuxième symposium MedEd de l'Institut suisse pour la formation médicale postgraduée et continue (ISFM). Bull Méd Suisses. 2015;96(45):1638-41.

2 La liste des lauréats 2015 est disponible dans l'article [1], p. 1640.

3 Recommandations de la FMH et de l'ISFM sur la mention de titres. www.fmh.ch/fr/formation-isfm.html

4 Un article complet à ce sujet paraîtra dans le prochain numéro du BMS 